\title{
Application of tool change fault in machining center based on PLC
}

\author{
Jin Ying Chen ,Li Juan Shi
}

School of mechanical and electrical engineering Beijing Polytechnic,beijing100042, China

vegachjy@163.com

\section{Keywords: Machining center;PLC;Electric Control;Fault}

Abstract. The fault phenomenon tool real machining center for tool in the tool magazine was studied when using the PLC method, the electric control system for fault diagnosis, solve the problems of fault in tool changing machining center. As a result, the efficiency of dealing with failures is improved. This method provides experience for the employees to solve this problem.

\section{Introduction}

Most of faults to CNC machines can be detected with the help of PLC procedures with alarm message displayed on the screen or not generated. Therefore, there are numerous studies on handling and settlement of faults to CNC machines with the help of PLC functions in China. However, most of studies focus on Fanuc system and Siemens system other than high-end systems of Huazhong, such as 818 System.

The thesis aims to study faults to tool change by manipulator of HNC-818 processing center by taking flexibility and convenience of PLC function as the breakthrough point so as to realize accurate and effective elimination of faults and recovery of machine functions based on further analysis of PLC function of $818 \mathrm{CNC}$ machine.

\section{PLC Structure of HNC-818 Machining Center}

PMC of HNC-818 CNC system makes use of built-in soft PLC to realize sequential control of CNC machine, which is compiled with ladder chart for PLC programming language according to users' practical demands for the machine. PLC user program of HNC-818 CNC system aims to realize online editing via the editing interface on the ladder chart or 【LADDER】, the special software for Huazhong ladder chart in the computer.

Huazhong ladder chart adopts circulated scanning mode. Initialization is to be performed at execution of the program; after that, all statuses as input are to be sent to input image register to sequential execution of user programs. Once a scanning cycle is completed, all results are to be transferred to output image register to control actual input of PLC, running in circles. PLC specifications for the system are as shown in Table 1.

Table 1: PLC Specifications for HNC-818 CNC System

\begin{tabular}{|c|l|l|}
\hline Serial No. & \multicolumn{2}{|c|}{ Items and Specifications } \\
\hline 1 & Specifications & HNC8 \\
\hline 2 & Programming language & Ladde STL \\
\hline 3 & Execution cycle of primary program & $1 \mathrm{~ms}$ \\
\hline 4 & Ladder chart & 5,000 lines \\
\hline 5 & Statement list & 10,000 lines \\
\hline 6 & Symbols & 1,000 \\
\hline
\end{tabular}




\section{PLC Controlled Tool Change Process for Bamboo Hat Tool Magazine}

As shown in Table 1, tool magazine for HNC-818 CNC machine belongs to bamboo hat tool magazine.

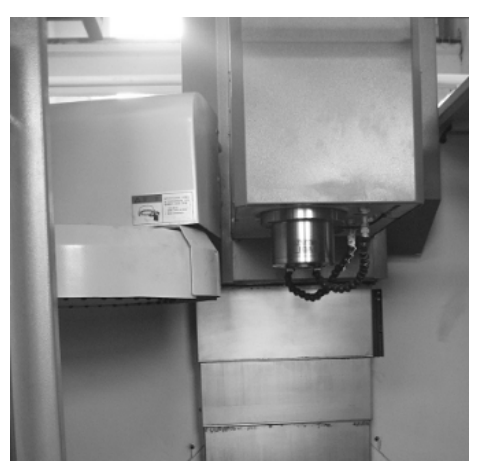

Figure 1: Tool Magazine Form

The tool magazine will be in parallel translation to the shaft in case of tool change.

Firstly, remove original tool on the shaft. Make the tool on the shaft move into the groove on the tool magazine; in such case, the shaft will move upward to disengage from the tool;

Secondly, install new tool on the shaft. Tool magazine will start to search the position of new tool number; when new tool is aligned with the lower part of the shaft, the shaft will move downwards to push new tool into the bore hole on the shaft for clamping.

Finally, tool magazine will return to original position to complete tool change for the tool magazine once new tool is installed.

Tool selection process for tool magazine is as shown in Figure 2.
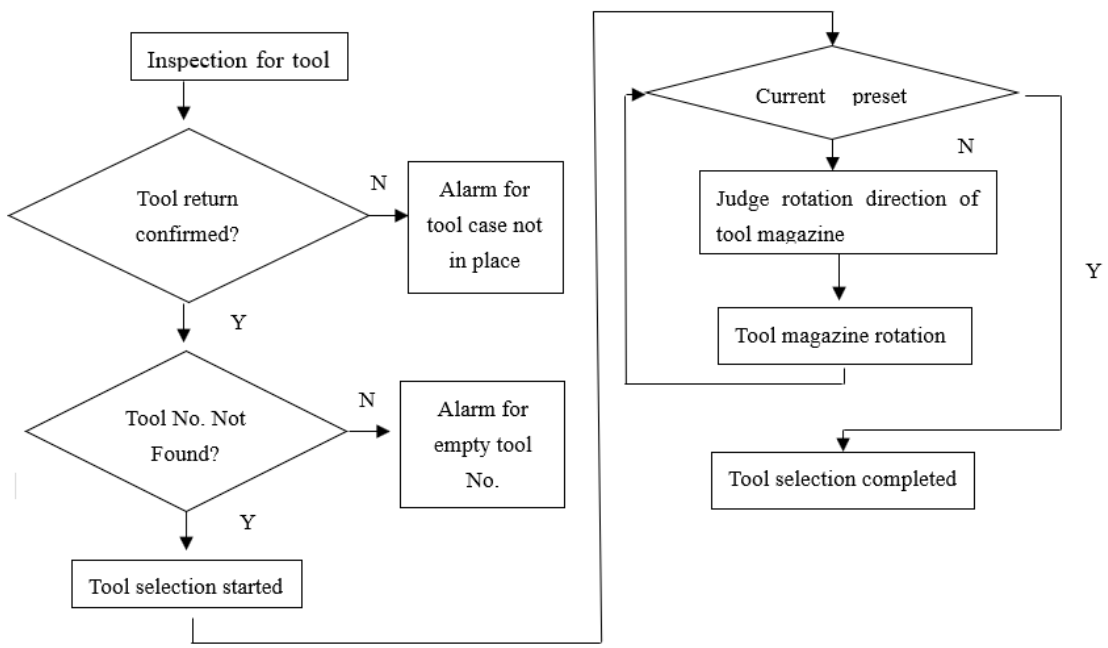

Figure 2: Flow Chart for Tool Selection of Tool Magazine 8

\section{Faults to Change of Bamboo Hat Tool Magazine and Solutions}

HNC-818 processing center is provided with a 24-position tool magazine. CNC will give out alarm for ATC NOT READY in case of tool change. Check the circuit and PLC control part of the machine in view of such problem to eliminate the fault.

In view of tool selection process as shown in Figure 2, tool magazine will not move, and will give out alarm message when the $\mathrm{CNC}$ system give instructions for tool change, for instance, 
M06T3; under such circumstance, it is applicable to check operation mode of the machine and status of air pump and tool magazine.

(1) Check operation mode of the machine

Make sure that the machine is in auto operation mode, and is not locked; make sure that input of tool change instructions is correct, and new tool to be installed is properly positioned in the tool magazine; there will be no fault is such item is proved to be correct through inspection.

(2) Check the air pump of the CNC machine

Check compressed air pressure inside the air pump of CNC machine; as checked in reference to operation instructions, the specified air pressure is $0.55 \mathrm{MP}$, which is within the range of 0.5MP-0.6MP for compressed air pressure inside the CNC machine. When compressed air pressure is below the specified value, tool magazine will not in operation during tool change due to inadequate pressure; however, the pressure in such case is up to the specified value; as checked, there is no fault to such item.

(3) Check initial status of tool magazine

Check initial status of tool magazine and status of the sensor with the help of PLC address diagnosis function provided by the CNC machine; check if input signal as sent to the PLC of CNC machine is correct. Enter the ladder chart "monitoring" interface in the "diagnosis" menu to check if there is any output signal; diagnosis interface of the ladder chart is as shown in Figure 3.

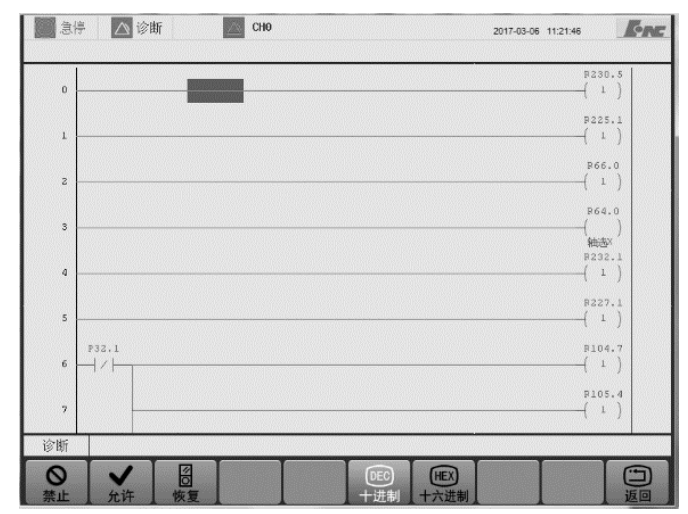

Figure 3: Ladder Chart Diagnosis Interface

For output module HIO-1021N as shown in Figure 4, output of rotation signal from tool magazine Y1.6 and Y1.7 is normal; as checked, tool magazine FWD and EXIT signal Y1.4 and output signal Y1.5 are normal, and PLC logic sequence of the system satisfies the specified conditions. As indicated by inspection for FWD in place signal X3.0 and EXIY signal X3.01 of tool magazine, tool clamp in place signal X3.2 and tool release in place signal X3.3 of the shaft based on $\mathrm{I} / \mathrm{O}$ interface as shown in Table 1, control signal circuit and elements are ok.

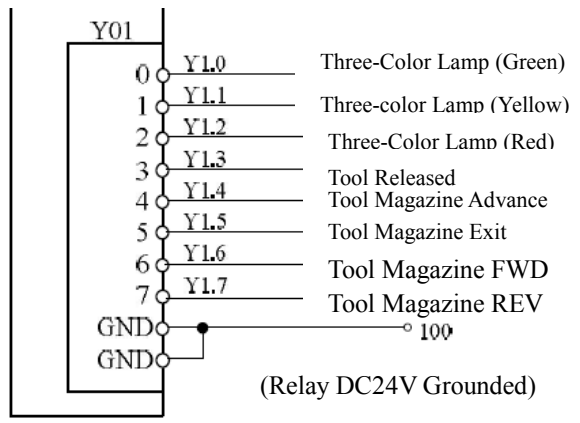

Figure 4: Output Module HIO-1021N 
Finally, check tool counting signal and intermediate relay of the tool magazine. There will be no problem if signal value of X1.7 is 1 , and problems with the circuit and tool magazine counting switch can be excluded; tool magazine counting PLC is as shown in Figure 5. In such case, tool pan cannot rotate, and tool magazine counting alarm message is displayed when FWD and REV switch on the tool magazine are pressed; further press FWD relay KA25 and REV relay KA26 to check FWD contactor KM5 and REV contactor KM6; it is discovered that KM5 and KM6 are unavailable for normal suction due to inadequate suction force. It is discovered through careful inspection that the problem is short circuit to the short-circuit ring. Tool magazine has recovered its normal operation after replacement of two contactors.

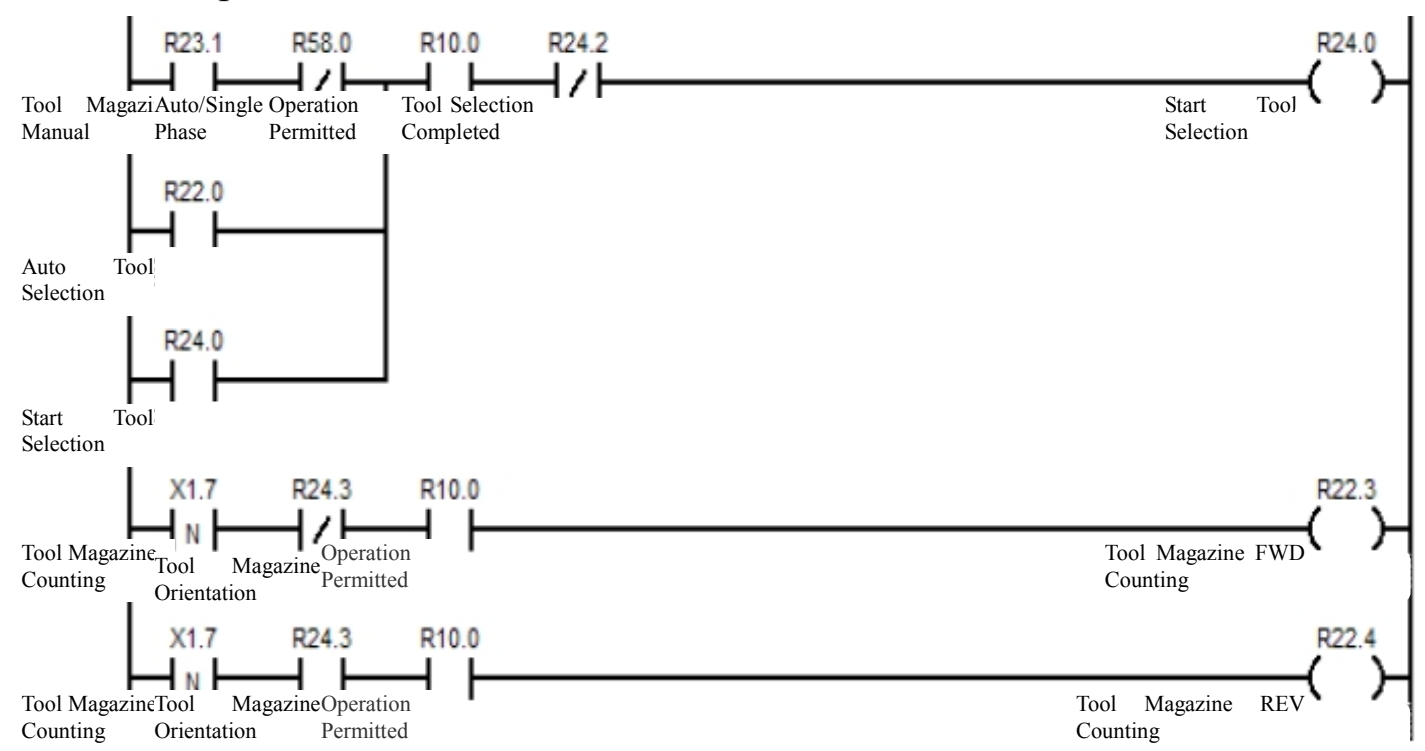

Figure 5: Tool Magazine Counting PLC

\section{Common Faults with Tool Change and Solutions}

(1) Abnormal tool magazine FWD/EXIT

Abnormal tool magazine FWD/EXIT is associated with relevant elements of the tool magazine, such as motor, relay and tool change proximity switch.

Firstly, check if power supply to the motor is normal, if the motor can rotate normally, and if there is any virtual connection to the relay line; check if there is any fusion or melting to the contact breaker;

Secondly, it is applicable to enter the CNC system of the machine to check operation status of PLC to judge if there is any fault point based on interfacing signal and PLC control of the machine;

Finally, check if tool change proximity switch is normal, if there is any virtual connection to lines of tool magazine ready signal and tool change signal, if signals are normal, if transmission mechanism and tool magazine dial is flexible and free of any jamming.

(2) Tool collision to CNC machine

It is essential to check solenoid valve and clamping signal from the shaft if there is any tool collision to the $\mathrm{CNC}$ machine in case of tool change.

Firstly, check tool clamping solenoid valve is normal when user program are proved to be free of any error; 
Secondly, enter the system PLC to check if such circle is normal based on I/O interface and PLC control of the machine; if there is any abnormality, it means that solenoid is in failure, and requires inspection and replacement.

Finally, check if coordinate $\mathrm{X}$ and $\mathrm{Y}$ is still available for shift when the shaft stop rotating due to loss of clamping signal from the shaft. In such case, it is applicable to modify PLC control procedures or adjust clamping switch to ensure normal press fitting.

\section{Conclusions}

The author has detected tool change fault during maintenance of the machine in a quick and accurate manner based on skillful command of principles and process for tool change of processing center. In particular, the author has discovered location of faults to the tool magazine through reference to and analysis of operation status of PLC ladder chart for careful analysis of underlying causes for the fault. This has effectively recovered tool change fault to the machine, and has provided a reliable guarantee for safe production.

\section{Acknowledgements}

This work was financially supported by Beijing Municipal Science and Technology Project (KM201610853003),Funded Research Subject (bgzyky 201723z).

\section{References:}

[1] E. N. Popov,A. L. Komkov,S. L. Ivanov. Modernization of the Control Systems of High-Frequency, Brush-Free, and Collector Exciters of Turbogenerators [J].Power Technology and Engineering,2016,Vol.50(4),pp.447-45.

[2] Qi-huai Chen,Qing-feng Wang,Tao Wang.Optimization design of an interior permanent-magnet synchronous machine for a hybrid hydraulic excavator[J].Frontiers of Information Technology \& Electronic Engineering,2015,Vol.16 (11),pp.957-968.

[3] Shih-Ming Wang,Chien-Da Ho,Po-Cheng Tsai,Chuntai Yen.Study of an efficient real-time monitoring and control system for BUE and cutter breakage for CNC machine tools[J].International Journal of Precision Engineering and Manufacturing,2014,Vol.15 (6), pp.1109-1115.

[4] Chen Jinying, Detection and Analysis Based on Thermal Error to BV75 CNC Milling Machine [J]. Modular Machine Tool \& Automatic Manufacturing Technique, 2015,(04):101-103+114.

[5] Jiang Kang, Zhang Teng and Feng Zhongxiang, An ANFIS-PID Control Strategy for Hanger of Semi-active Magneto-rheological Damper [J]. Modular Machine Tool \& Automatic Manufacturing Technique, 2016,(04):80-82+88.

[6] Chen Jinying, Guo Yong and Shi Lijuan, Measures and Approaches for Improvement of Teaching Quality of "CNC Milling Programming and Processing [J]. Journal of Beijing Vocational \& Technical Institute of Industry, 2016,(03):66-69. 
[7] Yu Rong, Sun Haoran and He Chaoming. Composite Fuzzy Adaptive PID Control of Servo System [J]. Modular Machine Tool \& Automatic Manufacturing Technique, 2017,(04):75-77+81.

[8] Zhang Jianghua. Control of Position of Chattering-Free Slip Form for DC Motor [J]. Modular Machine Tool \& Automatic Manufacturing Technique, 2017,(02):89-93.

[9] Shi Lijuan, Simulated Analysis and Diagnosis of Mismatched Shaft Speed [J]. Machine Tool \& Hydraulics, 2017,(04):202-204. 